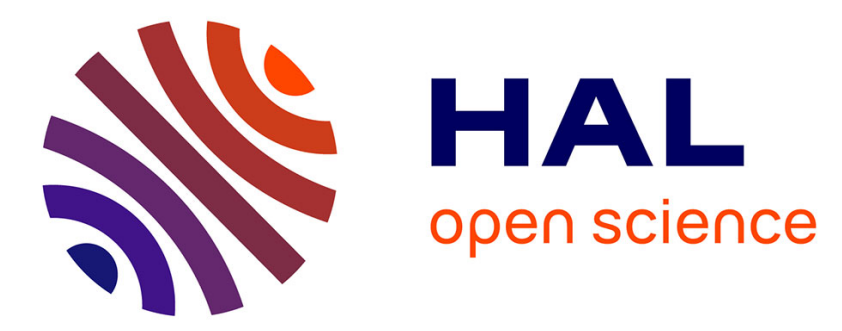

\title{
Infrared phase imaging using complex scattering media
}

Anwesh Bhattacharya, Pascal Berto, Valentina Krachmalnicoff, Ignacio

Izeddin, Gilles Tessier, Yannick de Wilde

\section{To cite this version:}

Anwesh Bhattacharya, Pascal Berto, Valentina Krachmalnicoff, Ignacio Izeddin, Gilles Tessier, et al.. Infrared phase imaging using complex scattering media. Infrared Sensors, Devices, and Applications XI, Aug 2021, San Diego, United States. pp.18, 10.1117/12.2593934 . hal-03444694

\section{HAL Id: hal-03444694 \\ https://hal.science/hal-03444694}

Submitted on 23 Nov 2021

HAL is a multi-disciplinary open access archive for the deposit and dissemination of scientific research documents, whether they are published or not. The documents may come from teaching and research institutions in France or abroad, or from public or private research centers.
L'archive ouverte pluridisciplinaire HAL, est destinée au dépôt et à la diffusion de documents scientifiques de niveau recherche, publiés ou non, émanant des établissements d'enseignement et de recherche français ou étrangers, des laboratoires publics ou privés. 


\title{
Infrared Phase Imaging using Complex Scattering Media
}

\author{
Anwesh Bhattacharya ${ }^{1}$, Pascal Berto ${ }^{2,3}$, Valentina Krachmalnicoff ${ }^{1}$, Ignacio Izeddin ${ }^{1}$, Gilles \\ Tessier $^{3}$, and Yannick De Wilde ${ }^{1}$ \\ ${ }^{1}$ ESPCI Paris, PSL University, CNRS, Institut Langevin, 1 Rue Jussieu, 75005 Paris, France \\ ${ }^{2}$ Université Paris Descartes, Sorbonne Paris Cité, Paris, France \\ ${ }^{3}$ Sorbonne Université, CNRS UMR7210, INSERM UMRS968, Institut de la Vision, Paris, \\ France
}

\begin{abstract}
Infrared imaging finds numerous applications in airborne measurements, structural monitoring, medical diagnostics, and spectroscopy. Long wave infrared (LWIR) radiation $(\lambda=8-14 \mu \mathrm{m})$ enables self-illuminated thermal imaging, and can uniquely identify different chemical species. The investigation of thermal emission control using plasmonic antenna devices, ${ }^{1}$ and the study of tunable free-form planar optics ${ }^{2}$ has motivated our development of a novel high-resolution thermal imaging technique. Speckle imaging has been successfully used to image optical intensity or phase through complex inhomogeneous scattering media - particularly at visible wavelengths, ${ }^{3}$ and recently in the infrared. ${ }^{4}$ Single-shot high-resolution images of the scattered light capture sufficient information to reconstruct images through opaque media and around corners with diffraction-limited resolution. In this context, we have developed a high-resolution broadband speckle imaging setup in the LWIR for phase reconstruction, using a thin scattering medium in front of an uncooled microbolometric camera. Our method utilizes the large angular memory effect of a thin scattering medium. Local phase gradients within the incoming beam produce distorted speckle images after scattering by the scatterer's surface. Local translation shifts between the speckle patterns are estimated by a fast diffeomorphic image registration algorithm to obtain a phase gradient map. Integrating this gradient map in 2-D finally yields the wavefront profile. We demonstrate infrared phase image reconstruction using our broadband LWIR speckle imaging methodology, which promises future applications in imaging through visually opaque objects like semiconductor circuits, solid-state nanoelectronics, and infrared optical components, for defect monitoring.
\end{abstract}

Keywords: Phase imaging, Infrared imaging, Wavefront sensing, Surface scattering, Complex media, Broadband imaging

\section{INTRODUCTION}

Long wavelength infrared (LWIR) radiation enables imaging of objects without any external illumination, solely due to their emitted thermal radiation, ${ }^{5}$ making the wavelength range between $8-14 \mu \mathrm{m}$ particularly relevant for thermal imaging. LWIR also coincides with a transmittance window of the earth's atmosphere, making it useful for atmospheric and space applications. ${ }^{6}$ Owing to the presence of the rotational-vibrational states of numerous chemical compounds and molecules in the LWIR, it is also named the IR spectroscopy fingerprint region.

Thermal sources usually exhibit no spatial and temporal coherence, and thus radiate over a broad angular range and spectrum. There has been recent success in controlling the thermal emission properties by means of surface structuration on polar and plasmonic materials, ${ }^{7,8}$ and it is thus very useful to characterize the IR emitters themselves. We are specifically interested in investigating the far-field thermal emission from IR nano-antennas in the LWIR, and reconstructing thermal wavefronts using single-shot imaging. Although IR holography appeared as early as $1969,{ }^{9}$ the requirement of having a reference beam and precise alignment of the reference and sample beam renders holographic wavefront detection cumbersome.

An alternative method employed in wavefront sensors in the visible region ${ }^{10}$ of the electromagnetic (EM) spectrum is to directly map the local wavevectors to a two-dimensional space and integrate them to obtain a

Further author information: (Send correspondence to anwesh@espci.fr) 
phase map. Direct wavefront measurement is compatible with broadband sources, and high-resolution wavefront sensing can give rise to quantitative phase and intensity imaging by giving access to the complex amplitude of the field. Visible wavefront sensing using scattering media has been shown to be able to encode the wavefront information in an intensity pattern: ${ }^{11}$ a small deformation in the output speckle in comparison to a reference speckle allows the reconstruction of the incident wavefront with high fidelity. ${ }^{3}$

In this paper, we report the development of a broadband speckle imaging setup in the LWIR for phase reconstruction, using a thin scattering medium in front of an uncooled microbolometric camera. Our setup allows us to qualitatively reconstruct test wavefronts in the LWIR, and opens the possibility for quantitative broadband phase imaging in the LWIR with an uncooled thermal camera.

\section{SPECKLE IMAGING}

A speckle is the mutual interference pattern between multiple wavefronts generated when light is reflected by or transmitted through a disordered material, whose surface roughness on the scale of an optical wavelength introduces fine-scale local random dephasing. ${ }^{12}$ The retrieval of the interference information encoded in speckle patterns was first proposed by A. Labeyrie in $1970,{ }^{13}$ leading to the development of speckle interferometry in astronomy. Once demonstrated, the ability to exploit speckle patterns to retrieve the encoded object information after transmission was widely adopted in diverse fields. Speckle imaging in the visible region of the EM spectrum has shown that it is possible to do single-shot image reconstruction of objects through a scattering medium. ${ }^{14}$ The spatial correlation of speckles allows to reconstruct spatial information, as the essential features of the object or scene are still preserved in the scattered speckle image. ${ }^{11}$
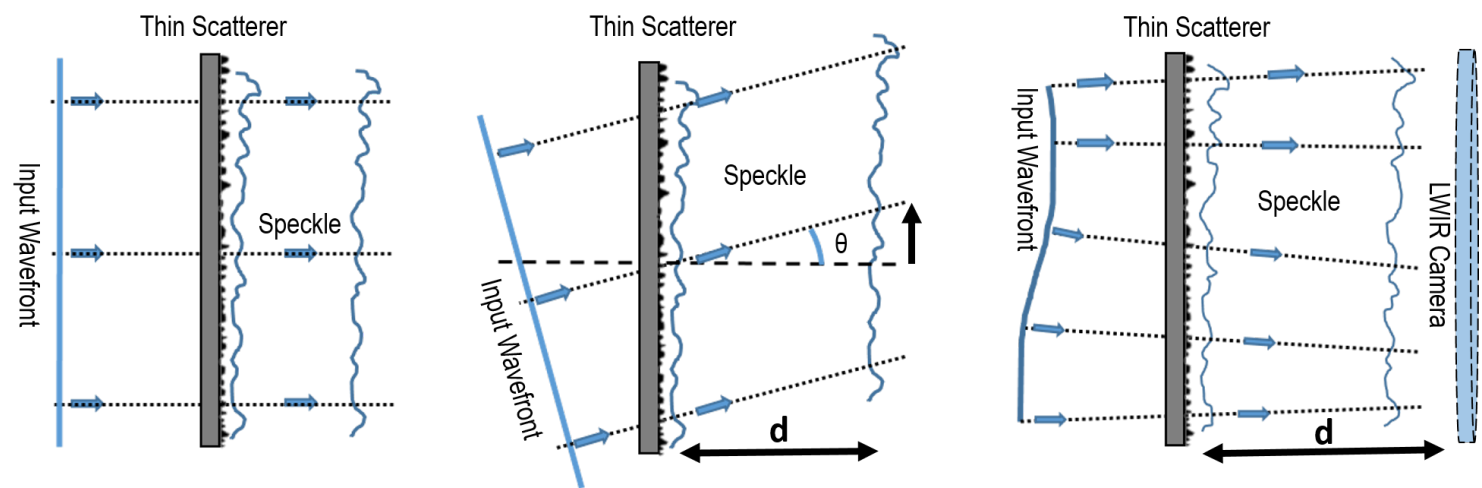

Figure 1: A speckle pattern is a unique fingerprint of the incident wavefront on a thin scattering medium. (a) A plane wavefront produces a speckle pattern after transmission through a thin surface scatterer. (b) A global tilt of the plane wavefront by the angle $\theta$ results in the same speckle pattern shifted by an amount $d \theta$ at a distance $d$, known as the 'memory effect'. (c) A local tilt in the wavefront changes the speckle pattern and results in a distorted speckle.

'Memory effect' is the phenomenon whereby a small global tilt in the input wavefront impinging on a thin scattering medium does not change the speckle pattern completely but only shifts the original speckle pattern in the transverse distance by an amount proportional to the tilt, as illustrated in Figure $1 .{ }^{15,16} \mathrm{~A}$ specific wavefront produces a corresponding unique speckle pattern, whose relative grain positions do not change if the wavefront globally shifts by an angle $\theta$. However, a local tilt in the wavefront produces a local deformation of the speckle pattern, which makes it possible to perform precise wavefront sensing as the speckle pattern is a unique fingerprint of the incident wavefront.

P. Berto et al. in 2017 demonstrated spatial and phase imaging of the wavefront from a broadband source through a thin scattering medium in the visible region of the EM spectrum, after a one-time speckle-to-wavefront calibration step. ${ }^{3}$ If we assume that $\theta$, the global tilt angle of the incident wavefront is small, then the optical path difference $\delta$ is related to the tilt angle as $\theta \simeq \tan \theta \simeq \sin \theta=\left\|\nabla_{\perp} \delta\right\|$. After the transmission of the wavefront 
through the scattering medium and formation of the speckle pattern, the displacement vector field of the speckle grains, $\mathbf{s}$, is proportional to the gradient of the optical path difference $(\delta)$ encountered by the beam:

$$
\nabla_{\perp} \delta=\frac{\nabla_{\perp} \varphi}{k_{0}} \simeq \frac{\mathbf{s}}{d}
$$

where, $d$ is the distance between the scattering medium and the speckle imaging plane of the camera, $\varphi$ is the phase of the wavefront, and $k_{0}=\frac{2 \pi}{\lambda}$ is the wavenumber. This equation allows us to obtain the phase of the wavefront by calculating the displacement $\mathbf{s}$ over the entire field. Several algorithms can calculate this displacement vector field. As shown in section 3, we use here the fast non-rigid image registration algorithm called diffeomorphic Demon's algorithm. ${ }^{17,18,19}$ The 2-D integration of the gradient of this displacement vector field then yields the reconstructed wavefront.

\section{EXPERIMENTAL SETUP \& RESULTS}

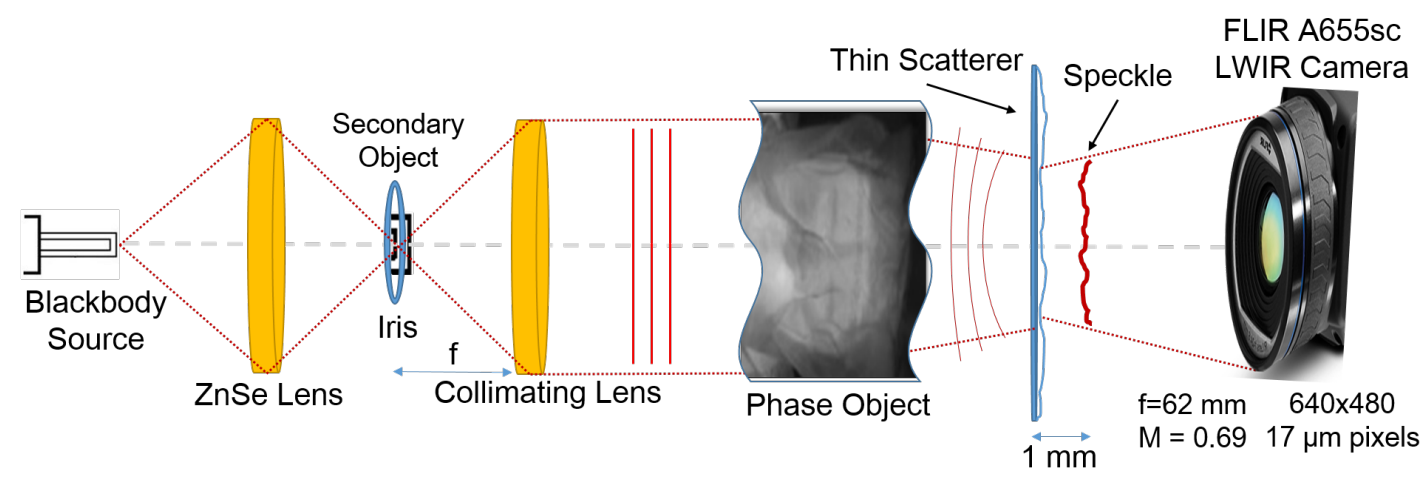

Figure 2: Experimental Setup for LWIR Speckle Imaging. A blackbody source produces a magnified image at the iris, where its spatial extent can be controlled, giving rise to a secondary object which is then collimated to produce a plane wavefront. The plane wavefront is incident on a phase object, which distorts the wavefront. The distorted wavefront is incident on a thin infrared surface scatterer which gives rise to a speckle after transmission. The speckle pattern is imaged by a FLIR A655sc LWIR uncooled microbolometric camera at a plane $1 \mathrm{~mm}$ away from the scatterer's surface.

In order to carry out speckle imaging of thermal wavefronts in the far field, we have developed a simple optical setup as shown in Figure 2. The basic components of the optical setup are an infrared source, an iris for spatial filtering, a Köhler illumination scheme followed by optics for collimating the incident beam, a scattering medium and an infrared camera. The blackbody source is a globar made of silicon carbide whose temperature can be raised above $1000 \mathrm{~K}$ by Joule heating. The lenses used for image formation and collimation are 1-inch Zinc Selenide lenses with anti-reflection coatings between 7-12 $\mu \mathrm{m}$. The source is imaged using a Zinc Selenide lens onto the aperture of the iris to serve as a secondary source whose spatial extent can be controlled by varying the aperture of the diaphragm. The mounted Iris is used as an aperture in the Köhler illumination part of the setup in order to control the spatial coherence of the source. A magnified image of the globar is formed on the Iris plane and this becomes the secondary source. The secondary source is then collimated using a Zinc Selenide lens placed one focal length away from the Iris plane to produce an image of the secondary source at infinity. This parallel beam is incident on the scattering medium, where it undergoes surface scattering. The transmitted light forms a speckle due to the inhomogeneous surface roughness of the scattering medium's exit surface. The speckle formed $1 \mathrm{~mm}$ away from the scattering medium is imaged using a close-up lens of focal length $f=62$ mm and magnification $M=0.69$ onto the sensor of a FLIR A655sc uncooled microbolometric LWIR camera. The imaging setup is therefore, a broadband uncooled thermal speckle imaging configuration.

In our imaging methodology, we typically capture 1000 frame movies at $25 \mathrm{~Hz}$ in background, reference and sample configurations, and average them before processing. An image of the background thermal radiation 
is captured by placing all the optical elements, with the globar source off. This image of the environment is subtracted from all subsequent images. Then, a reference image of the speckle is acquired by switching on the globar and capturing the speckle that is created due to the incident collimated beam on the scattering medium. Finally, a sample image is acquired by placing a phase object between the collimating lens and the scattering medium. Here, we use a Zinc Selenide lens of focal length, $f=1 \mathrm{~m}$, as a known wavefront modifier to test the viability of wavefront reconstruction in the LWIR. The placement of this sample lens between the collimating lens and the scattering medium distorts the plane wavefront into a converging wavefront that has a focus 1 metre away.

The speckle shift between the reference speckle and the sample speckle is computed by using the non-rigid image matching technique of diffeomorphic Demon's algorithm. The latter is an iterative second-order gradient descent algorithm that seeks to minimize the mean squared difference function between the reference and sample images. In this case, it enables an estimation of a diffeomorphism between the two speckles. The reference image is considered as a static image grid having effectors known as Demons, which alter a deformable grid of the sample image to diffuse through the contours of the static reference image, ultimately trying to match the sample image to the reference image. The deformations effected by the Demons are then used to compute a displacement field using a Gaussian filter which is applied to the whole field at each iteration. The algorithm employs a multi-scale pyramid approach in considering all voxels of the images, with larger number of iterations at the coarser scales and only a few iterations at the finer scales to improve the speed of the algorithm and robustness to initial positions. The computed displacement vector field is then integrated in the Fourier domain to obtain the reconstructed wavefront. It is critical to know the proportionality coefficient between the local wavevector and the displacement vector map. This can be effectively calibrated by knowing the distance between the scattering medium and the imaging plane of the camera, which in our case is $1 \mathrm{~mm}$ away.
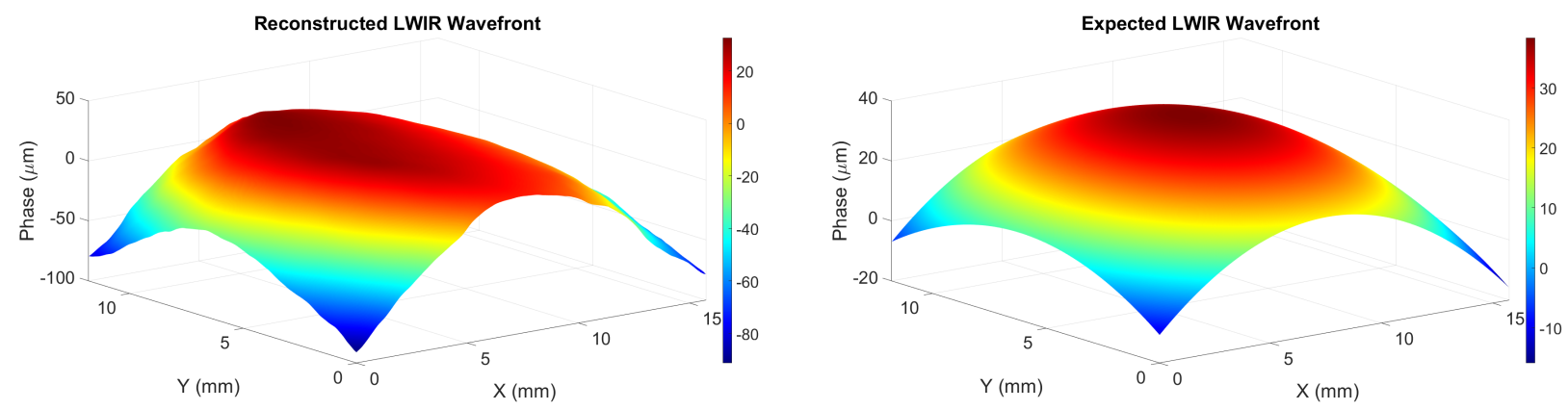

Figure 3: LWIR Wavefront Reconstruction. Experimentally reconstructed (left) \& ideal expected (right) wavefronts when a Zinc Selenide lens with $f=1 \mathrm{~m}$ is placed as a phase sample in the setup shown in Figure 2 . The field of view of the camera close-up lens at the speckle imaging plane is $16 \mathrm{~mm}$ x $12 \mathrm{~mm}$.

As shown in Figure 3, the reconstructed wavefront displays the expected spherical shape, as shown in the simulated wavefront. This qualitative similarity of the reconstructed wavefront to the ideal expected wavefront is evident from the symmetric spherical profile of the reconstructed wavefront whose phase values match those of the expected wavefront. While improvement of the optical setup is still possible, this promising result opens up the avenue towards quantitative wavefront reconstruction in the LWIR.

\section{CONCLUSION}

We have developed a simple broadband phase imaging methodology in the long wave infrared, and demonstrated the reconstruction of the wavefront of a phase modifying object using a blackbody source and a thin scattering medium. By taking advantage of the memory effect of a thin scattering medium and the efficient diffeomorphic Demon's algorithm, the proposed LWIR wavefront reconstruction method is compact, fast, and also avoids reconstruction artifacts due to the uniqueness of the speckle patterns. The wavefront reconstruction methodology employs speckle imaging through a thin infrared scattering medium, and reinforces the notion that speckle imaging techniques can be transposed to different wavelength regions of the EM spectrum. 
In the future, we seek to improve our phase reconstruction experiment to perform precise quantitative phase reconstruction using known phase samples, and subsequently imaging visually opaque samples like semiconductor circuits, solid-state nanoelectronics, and IR optics for wavefront sensing and defect monitoring.

\section{ACKNOWLEDGMENTS}

This project has received funding from the European Union's Horizon 2020 research and innovation programme under the Marie Skłodowska-Curie grant agreement No. 754387. This research is also supported by LABEX WIFI (Laboratory of Excellence within the French Program "Investments for the Future") under references ANR-10-LABX-24 and ANR-10-IDEX-0001-02 PSL.

\section{REFERENCES}

[1] Abou-Hamdan, L., Li, C., Haidar, R., Krachmalnicoff, V., Bouchon, P., and Wilde, Y. D., "Hybrid modes in a single thermally excited asymmetric dimer antenna," Optics Letters 46, 981-984 (Mar. 2021).

[2] Berto, P., Philippet, L., Osmond, J., Liu, C. F., Afridi, A., Montagut Marques, M., Molero Agudo, B., Tessier, G., and Quidant, R., "Tunable and free-form planar optics," Nature Photonics 13, 649-656 (Sept. 2019).

[3] Berto, P., Rigneault, H., and Guillon, M., "Wavefront sensing with a thin diffuser," Optics Letters 42, 5117 (Dec. 2017).

[4] Divitt, S., Gardner, D. F., and Watnik, A. T., "Imaging around corners in the mid-infrared using speckle correlations," Optics Express 28, 11051 (Apr. 2020).

[5] Socolinsky, D., Wolff, L., Neuheisel, J., and Eveland, C., "Illumination invariant face recognition using thermal infrared imagery," in [Proceedings of the 2001 IEEE Computer Society Conference on Computer Vision and Pattern Recognition. CVPR 2001], 1, I-I (Dec. 2001).

[6] Hackwell, J. A., Warren, D. W., Bongiovi, R. P., Hansel, S. J., Hayhurst, T. L., Mabry, D. J., Sivjee, M. G., and Skinner, J. W., "LWIR/MWIR imaging hyperspectral sensor for airborne and ground-based remote sensing," in [Imaging Spectrometry II], 2819, 102-107, International Society for Optics and Photonics (Nov. 1996).

[7] Greffet, J.-J., Carminati, R., Joulain, K., Mulet, J.-P., Mainguy, S., and Chen, Y., "Coherent emission of light by thermal sources," Nature 416, 61-64 (Mar. 2002).

[8] Laroche, M., Arnold, C., Marquier, F., Carminati, R., Greffet, J.-J., Collin, S., Bardou, N., and Pelouard, J.-L., "Highly directional radiation generated by a tungsten thermal source," Optics Letters 30, 2623-2625 (Oct. 2005).

[9] Chivian, J. S., Claytor, R. N., and Eden, D. D., "Infrared holography at 10.6 $\mu \mathrm{m}, "$ Applied Physics Letters 15, 123-125 (Aug. 1969).

[10] Rueckel, M., Mack-Bucher, J. A., and Denk, W., "Adaptive wavefront correction in two-photon microscopy using coherence-gated wavefront sensing," Proceedings of the National Academy of Sciences 103, 1713717142 (Nov. 2006).

[11] Katz, O., Heidmann, P., Fink, M., and Gigan, S., "Non-invasive single-shot imaging through scattering layers and around corners via speckle correlations," Nature Photonics 8, 784-790 (Oct. 2014).

[12] Goodman, J. W., [Speckle Phenomena in Optics: Theory and Applications], Roberts and Company Publishers (2007).

[13] Labeyrie, A., "Attainment of Diffraction Limited Resolution in Large Telescopes by Fourier Analysing Speckle Patterns in Star Images," Astronomy and Astrophysics 6, 85 (May 1970).

[14] Sahoo, S. K., Tang, D., and Dang, C., "Single-shot multispectral imaging with a monochromatic camera," Optica 4, 1209 (Oct. 2017).

[15] Feng, S., Kane, C., Lee, P. A., and Stone, A. D., "Correlations and Fluctuations of Coherent Wave Transmission through Disordered Media," Physical Review Letters 61, 834-837 (Aug. 1988).

[16] Freund, I., Rosenbluh, M., and Feng, S., "Memory Effects in Propagation of Optical Waves through Disordered Media," Physical Review Letters 61, 2328-2331 (Nov. 1988). 
[17] Thirion, J.-P., "Image matching as a diffusion process: An analogy with Maxwell's demons," Medical Image Analysis 2, 243-260 (Sept. 1998).

[18] Vercauteren, T., Pennec, X., Perchant, A., and Ayache, N., "Diffeomorphic demons: Efficient nonparametric image registration," NeuroImage 45, S61-72 (Mar. 2009).

[19] Pennec, X., Cachier, P., and Ayache, N., "Understanding the "Demon's Algorithm": 3D Non-rigid Registration by Gradient Descent," in [Medical Image Computing and Computer-Assisted Intervention - MICCAI'99], Goos, G., Hartmanis, J., van Leeuwen, J., Taylor, C., and Colchester, A., eds., 1679, 597-605, Springer Berlin Heidelberg, Berlin, Heidelberg (1999). 